\section{OPEN ACCESS}

Edited by:

Douglas Hawkins,

Seattle Children's Hospital, USA

Reviewed by:

David Loeb,

Johns Hopkins University, USA

Gene P. Siegal,

University of Alabama at Birmingham,

USA

*Correspondence:

Liliana Vasquez

lilianavasq@gmail.com

Specialty section:

This article was submitted to

Pediatric Oncology,

a section of the journal

Frontiers in Oncology

Received: 16 September 2015

Accepted: 21 January 2016

Published: 02 February 2016

Citation:

Vasquez $L$, Tarrillo F, Oscanoa $M$

Maza I, Geronimo J, Paredes G, Silva JM and Sialer L (2016) Analysis of Prognostic Factors in High-Grade

Osteosarcoma of the Extremities in Children: A 15-Year Single-Institution

Experience.

Front. Oncol. 6:22.

doi: 10.3389/fonc.2016.00022

\title{
Analysis of Prognostic Factors in High-Grade Osteosarcoma of the Extremities in Children: A 15-Year Single-Institution Experience
}

Liliana Vasquez ${ }^{*}$, Fanny Tarrillo', Monica Oscanoa', Ivan Maza', Jenny Geronimo', Gloria Paredes ${ }^{1}$, Jose María Silva ${ }^{2}$ and Luis Sialer ${ }^{2}$

'Division of Pediatric Oncology, Rebagliati Hospital, Lima, Peru, ${ }^{2}$ Department of Orthopedics, Rebagliati Hospital, Lima, Peru

Background: The aim of this retrospective study was to define clinical and pathological features and prognostic factors among children and adolescents diagnosed with highgrade osteosarcoma of the extremities.

Methods: A total of 73 patients younger than 18 years diagnosed with primary osteosarcoma of the extremities between January 1998 and December 2013 were retrospectively evaluated. Prognostic factors, such as age, gender, primary tumor site, alkaline phosphatase and lactate dehydrogenase levels, metastatic disease, pathological fracture, histological response, and surgery type, were analyzed to evaluate their effects on overall survival (OS) and event-free survival (EFS).

Results: At a median follow-up of 30 months (1.5-152), OS and EFS at 5 years were $64.5 \pm 8.1$ and $48.5 \pm 8.7 \%$ for patients with localized disease; and $16.2 \pm 7.9$ and $14.4 \pm 7.3 \%$ for patients with initial metastatic disease, respectively. In patients with localized disease, conservative surgery was performed on 22 of 46 patients (43.5\%), and there was no significant difference in survival rates among patients who had conservative vs. radical surgery $(p=0.65)$. Although tumor size $(>12 \mathrm{~cm})$ was significant prognostic factor in univariate analysis; multivariate analysis identified elevated levels of alkaline phosphatase $(p=0.033)$ and poor response to neoadjuvant chemotherapy $(p<0.001)$ only as independent prognostic factors. Age, histological type, pathological fracture, and primary tumor site did not significantly affect prognosis.

Conclusion: Initial elevated presence of alkaline phosphatase in serum and poor histological response after neoadjuvant chemotherapy were significant factors for unfavorable prognosis. It is necessary to optimize staging and treatment intensification to improve survival rates, especially among patients with metastasis at initial presentation.

Keywords: osteosarcoma, children, prognostic factors

\section{INTRODUCTION}

Osteosarcoma is the most frequent malignant bone tumor at pediatric age. Surgical removal of the tumor remains the most important treatment; however, survival rates have increased due to the advent of chemotherapy (1-3). In prospective studies, 5-year event-free survival (EFS) rates between 55 and $75 \%$ have been reported (4-6), and recurrence occur approximately $30-40 \%$ of patients with 
localized disease, in spite of complete surgical resection of primary tumor and an intense regimen of chemotherapy (7). These findings reveal an important need to clarify prognostic factors for recurrence or poor survival.

Currently, several studies have already identified clinical or pathological features associated with worse outcome. Some published data contain a reduced but homogeneous number of cases of limited statistical significance $(8,9)$. Moreover, multicenter research studies differ in data collection and therapeutical schemes (10-13), which makes it difficult to compare and reveals contradictory results.

In our region, there are no published studies defining prognostic factors for osteosarcoma, which is essential to improve the development of new and adapted strategies according to risk groups so as to improve survival rates.

The aim of the present study is to define clinical and pathological features and prognostic factors related to survival rates among pediatric patients diagnosed with high-grade osteosarcoma of the extremities in our country.

\section{MATERIALS AND METHODS}

\section{Patients}

Seventy-three patients diagnosed with primary high-grade osteosarcoma of the extremities (both localized and metastatic) were retrospectively evaluated. All patients were treated at the Pediatric Oncology Unit of the Rebagliati Hospital in Lima, Peru between January 1998 and December 2013. All patients who received chemotherapy and surgical treatment in our hospital were included.

\section{Diagnostic Methods and Staging}

Plain X-rays and CT scans or MRI of the primary tumor were performed at the time of diagnosis, prior to surgery, and at the end of therapy. Chest CT scan and bone scintigraphy were performed to detect distant metastases. All patients underwent confirmatory biopsy (open or by tru-cut needle) in our institution, and the diagnosis was confirmed by conventional light microscopy (histology). All cases of high-grade osteosarcoma were included (conventional, telangiectatic, fibroblastic, and small cell subtype). Patients with low grade tumors, such as parosteal osteosarcoma, were excluded.

\section{Systemic Treatment}

After diagnosis and staging, the patient started neoadjuvant chemotherapy. Seventy-one patients $(97.3 \%)$ received chemotherapy based on SEOP-95 consisted of high-dose methotrexate at $12 \mathrm{~g} / \mathrm{m}^{2}$ per cycle with leucovorin rescue for 11 cycles, adriamycin at $75 \mathrm{mg} / \mathrm{m}^{2}$ per cycle for 6 cycles, cisplatin at $120 \mathrm{mg} / \mathrm{m}^{2}$ per cycle for 2 cycles. Additionally, ifosfamide at $9 \mathrm{~g} / \mathrm{m}^{2}$ was used for six cycles. Length of chemotherapy treatment was 37 weeks. Definitive surgery was scheduled on week 15. A chemotherapy regimen without methotrexate (OS99) was used in two patients due to renal toxicity secondary to methotrexate (after two cycles on the previous regimen). Neoadjuvant chemotherapy treatment was lengthened $(n=4)$ or shortened $(n=2)$ in six patients $(8.3 \%)$ due to problems related to social security coverage or availability of prosthesis.

\section{Local Treatment}

The first definitive surgical treatment was documented as conservative (resection of primary tumor) with or without endoprosthesis $(n=31)$; or radical (amputation or disarticulation) $(n=42)$. Surgery type and reconstruction were decided according to tumor site and extension, patient's age, and presence of compromised neurovascular structures.

\section{Statistical Analysis}

Data about time from first symptoms to beginning of therapy, age, gender, primary tumor site, initial alkaline phosphate (AP) and lactate dehydrogenase (LDH) levels, metastatic disease at onset, presence of pathological fractures, histological response or necrosis after completion of neoadjuvant chemotherapy ( $<90 \%$ and more or equal to $90 \%)(14,15)$, and type of surgery were evaluated. SPSS 22.0 Statistics was used (SPSS Corp., USA). Overall survival (OS) was defined as the time from diagnosis to death from any cause and EFS was defined as the time from diagnosis to treatment failure, secondary neoplasm, or death, whichever came first. Patients who did not suffer an event were sensored at the time of last follow-up. OS and EFS rates were analyzed, according to Kaplan-Meier curves. A multivariate Cox proportional hazards regression analysis was performed to determine which parameters were significant. A 95\% confidence interval level was used; being a $p<0.05$ was considered significant.

\section{Ethical Considerations}

Our study was not set up as a study or research project; hence, we did not seek informed consent or ethical committee approval due to it does not report on primary research. Absolutely, all data analyzed were collected as part of routine diagnosis and treatment, and all patients were diagnosed and treated according to institutional guidelines and agreements. All laboratory tests (as well as recording all other variables included in our analysis) are essential for confirming diagnosis and classifying patients, and they are done for each patient without fail and as part of routine care. Moreover, this paper does not report on the use of experimental therapies. We looked retrospectively at outcomes for a long-term cohort of patients treated as a process of an audit/ evaluation, so as to improve our quality of care.

\section{RESULTS}

\section{Patient Characteristics}

A total of 73 patients under 18 years of age were included. Patient characteristics are listed in Table 1. The mean age at diagnosis was 14 years (range, 5-17 years). Sixty-six percent of patients were male (45 patients). Average length of follow-up period was 30 months (1.5-152 months). There was metastatic disease at initial presentation in 27 patients (37\%), being the lungs the most commonly affected organ (24 patients), followed by bone metastases ( 3 patients). Latency time from manifestation of the first symptoms and the beginning of treatment was 4.3 months on average, with a range of $0.3-19$ months.

In the localized group, conservative surgery was performed in 22 out of 46 patients (47.8\%), and no significant difference was 
TABLE 1 | Demographic and clinical characteristics $(N=73)$.

\begin{tabular}{lc}
\hline Characteristic & Number of patients (\%) \\
\hline Male $(n, \%)$ & $45(61.6 \%)$ \\
Age at diagnosis (years) & \\
Mean & 14 \\
Range & $5-17$ \\
Histological subtype & \\
Osteoblastic & $52(71.3 \%)$ \\
Chondroblastic & $12(16.4 \%)$ \\
Telangiectatic & $5(6.8 \%)$ \\
Fibroblastic & $3(4.1 \%)$ \\
Other & $1(1.4 \%)$ \\
Location of tumor & \\
Distal femur & $33(45.2 \%)$ \\
Proximal tibia & $15(20.5 \%)$ \\
Proximal femur & $10(13.7 \%)$ \\
Humerus & $9(12.3 \%)$ \\
Fibula & $6(8.3 \%)$ \\
Distant metastases at onset & \\
Yes & $27(37 \%)$ \\
No & $46(63 \%)$ \\
Histological response $(n=40)$ & $21(52.5 \%)$ \\
$\geq 90 \%$ & $19(47.5 \%)$ \\
$<90 \%$ & $58(79.5 \%)$ \\
Elevated serum AP & $57(78.1 \%)$ \\
Elevated serum LDH & \\
Type of surgery & $31(42.5 \%)$ \\
Amputation & $42(57.5 \%)$ \\
Tumor size $(n=32)$ & \\
$>12$ cm & $25(78.1 \%)$ \\
\hline
\end{tabular}

observed in survival rates related to type of surgery $(p=0.65)$. In both groups (localized and metastatic), radical surgery (amputation or disarticulation) was performed in 42 cases, and limb-preservation surgery (with or without endoprosthesis) was done on 31 patients. Patients with localized disease had better chances of having conservative surgery compared to those with metastatic disease at presentation $(p=0.003)(47.8$ vs. $25.9 \%$ ).

Twenty-three patients (31.5\%) had recurrent disease (3 local and 20 metastatic). In 19 cases $(82.6 \%)$, recurrence occurred within the first 3 years of diagnosis. Initial treatment was surgical if recurrence was local (three patients), with amputation in two cases and tumor resection in one patient. These patients received second-line chemotherapy (etoposide or carboplatin based). Only the patient who had tumor resection surgery had no evidence of disease for 42 months, whereas the other two died from progressive disease. Treatment in case of metastatic recurrence (20 patients) was surgical in 13 cases (65\%) (lung metastasectomy, bilateral, when possible) or second-line systemic chemotherapy alone in 7 cases (35\%) in case surgery was not possible (due to irresectable tumor or multiple lung nodules), presence of distant lesions in the liver and brain, or refusal to surgery. Nine of 20 patients (45\%) died from progressive disease. In two cases of metastatic pulmonary relapse, after surgical resection of lung metastases, high-dose chemotherapy was administered and autologous stem cell transplant was performed. In one of those two cases, the patient died from
TABLE 2 | Summary of univariate Cox proportional hazards model for overall survival.

\begin{tabular}{|c|c|c|c|c|}
\hline \multirow[t]{2}{*}{ Variables } & $\begin{array}{c}\text { Univariate } \\
\text { LRT }\end{array}$ & \multirow[t]{2}{*}{$\begin{array}{c}\text { HR } \\
(95 \% \mathrm{Cl})\end{array}$} & $\begin{array}{c}\text { Multivariate } \\
\text { LRT }\end{array}$ & \multirow[t]{2}{*}{$\begin{array}{c}\text { HR } \\
(95 \% \mathrm{Cl})\end{array}$} \\
\hline & $p$-Values & & $p$-Values & \\
\hline Male gender & 0.78 & $1.14(0.44-2.94)$ & & \\
\hline Age, $<10$ years & 0.09 & $3.73(0.42-2.47)$ & & \\
\hline $\begin{array}{l}\text { Histological } \\
\text { subtype }\end{array}$ & 0.96 & $1.02(0.15-6.64)$ & & \\
\hline $\begin{array}{l}\text { Location of } \\
\text { tumor }\end{array}$ & 0.57 & $0.77(0.31-1.94)$ & & \\
\hline $\begin{array}{l}\text { Initial } \\
\text { metastases }\end{array}$ & $<0.01$ & $4.88(3.48-40.57)$ & $<0.01$ & $\begin{array}{c}4.6 \\
(3.71-38.93)\end{array}$ \\
\hline Necrosis $>90 \%$ & $<0.01$ & $0.08(0.02-0.31)$ & $<0.01$ & $\begin{array}{c}0.13 \\
(0.06-0.28)\end{array}$ \\
\hline $\begin{array}{l}\text { Tumor size } \\
>12 \mathrm{~cm}\end{array}$ & $<0.01$ & 4.01 (2.43-8.12) & 0.058 & $\begin{array}{c}3.5 \\
(0.92-9.39)\end{array}$ \\
\hline Initial raised ALP & 0.027 & $3.90(1.11-13.71)$ & 0.033 & $\begin{array}{c}3.1 \\
(1.10-5.27)\end{array}$ \\
\hline $\begin{array}{l}\text { Initial raised } \\
\mathrm{LDH}\end{array}$ & 0.18 & $2.13(0.63-6.67)$ & & \\
\hline $\begin{array}{l}\text { Pathological } \\
\text { fracture }\end{array}$ & 0.30 & $1.86(0.56-6.22)$ & & \\
\hline
\end{tabular}

LRT, likelihood ratio test; HR, hazards ratio; $C l$, confidence interval.

Significant variables ( $p$ values $<0.05$ ) are in bold.

progressive of disease, and the other had a complete remission with a follow-up of 6 years.

\section{Prognostic Factors for Overall Survival}

Univariate and multivariate analysis of factors predicting treatment failure is shown in Table 2. An initial elevated serum level of AP $(p=0.027)$, poor histological response (necrosis of $<90 \%$ ) $(p<0.01)$, large tumor size $(>12 \mathrm{~cm})(p<0.01)$, and metastatic disease $(p<0.01)$ was statistically significant for worse survival. All these variables except tumor size remained significant on multivariate analysis.

Gender, age, initial LDH levels, presence of pathological fracture, histological subtype (osteoblastic, chondroblastic, telangiectatic or fibroblastic), latency time of symptoms, primary site tumor, and type of surgery were not significant for survival in univariate or multivariate analysis.

Surgical margins were compromised in two patients $(2.8 \%)$ who had metastasis at initial presentation and underwent limb amputation after neoadjuvant chemotherapy. Both died due to progressive disease. No statistical analysis was performed due to small number of patients.

Some patients had an alteration in the neoadjuvant chemotherapy sequence (it was either longer or shorter) due to problems with health insurance or with availability of prosthesis. There was no statistical difference with respect to those who received standard therapy.

\section{OS and EFS}

Overall survival and EFS at 5 years was $64.5 \pm 8.1$ and $48.5 \pm 8.7 \%$ for the localized group, and $16.2 \pm 7.9$ and $14.4 \pm 7.3 \%$ for the metastatic group, respectively (Figures 1 and 2). 


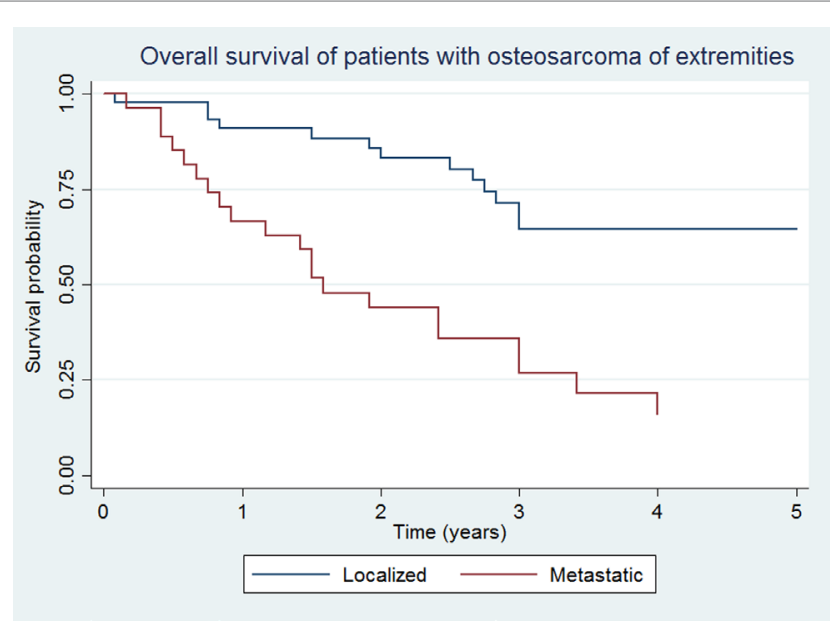

FIGURE 1 | Overall survival of patients with localized and metastatic osteosarcoma of extremities $(N=76)$

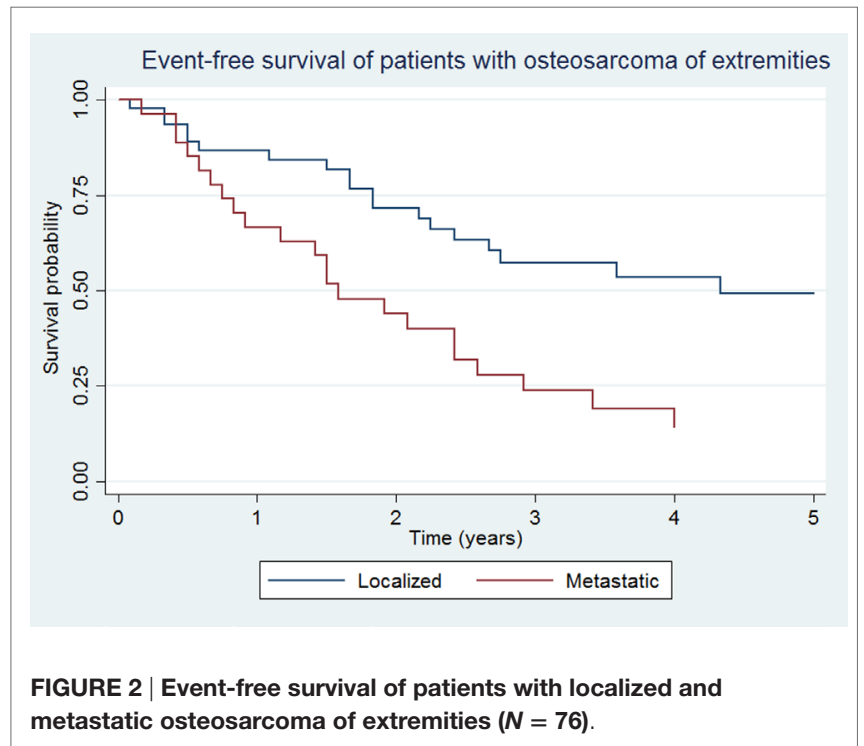

\section{DISCUSSION}

Identifying prognostic factors in osteosarcoma patients is vital to define risk groups. A number of clinical and pathological variables, such as histological subtype, age, gender, elevated levels of AP or LDH, and genetic variations have been previously studied with prognostic significance, but often with contradictory results due to lack of homogeneity in analysis and methods. In a systematic revision of prognostic factors for osteosarcoma, it was found that histological response, presence of metastasis, and primary tumor size and site were important prognostic factors (16).

Our study found considerably higher incidence of clinically detectable metastatic disease at initial presentation when compared to expected rates in developed countries (10-20\%) (17-19), or even when compared to other Latin American studies, like those done in Brazil $(20.8 \%)(5,20,21)$, being almost $40 \%$. This is probably due to delay in diagnosis and advanced disease at the moment of diagnosis, and it turns out to be the first cause of mortality among these patients, which makes it a significant prognostic factor, as reported by prior studies $(5,6)$.

As previously described (17), presence of pathological fractures ranges from 7 to $18 \%$ (in our study 14\%) and, while it did not proof to be prognostic as reported earlier (22), it is an important indicator of late diagnosis in our cases.

Age has been identified as a prognostic factor for osteosarcoma. Patients under the age of 10 (18) or 12 years (9) have poorer survival rates in some reports, which suggest aggressive behavior of this disease in small children. Nevertheless, more recent studies have failed to confirm the latter $(13,19)$, and we have also not observed this in our study. In a recent study, patients with age above 40 years also carried a dismal prognosis (23).

Gender was not prognostic, as has already been mentioned in European $(13,18)$ and American report studies (9). However, Brazilian (20) and Scandinavian (24) studies have included observations about female gender having a better prognosis.

The present study has not found any significant difference in survival rates according to histological subtype, as previously reported (25). According to other authors, there is evidence of lower risk of recurrence in fibroblastic and telangiectatic subtypes (26). It has also previously been mentioned that the chondroblastic subtype could be the one with the best prognosis (27). Furthermore, histological response has been more favorable in fibroblastic and telangiectatic groups, and less favorable in the chondroblastic group (28).

Prior studies have revealed that surgery type (radical vs. conservative) in patients with high-grade non-metastatic osteosarcoma does not affect survival or local recurrence rates (29-31). This is supported by our study, where no significant difference was found in both groups. In a study conducted by Bacci et al., 560 non-metastatic osteosarcoma patients were examined, and no variation in survival rates was found when considering surgery types. The percentage of conservative surgery, even though it has increased over the years and is currently at $20 \%$ of total operations, is still lower than the rates published by other groups (5).

Degree of necrosis as histological response to neoadjuvant chemotherapy is currently considered the most important prognostic factor in patients with high-grade osteosarcoma, according to reports in previous studies $(5-7,32)$. The percentage of patients who have a favorable response ( $\geq 90 \%)$ goes from 50 to $60 \%$ (32), whereas, it was $52.5 \%$ in our study.

The prognostic value of AP in osteosarcoma has previously been reported $(9,31,32)$ and has been confirmed in our study. Bacci et al. have reported that initial values of more than four times the normal level are linked to lower EFS rates (33).

Event-free survival rates among osteosarcoma patients worldwide are between 55 and 75\%, which has not improved significantly in recent years. Studies in developed countries mention survival rates of $70-75 \%$ in localized disease $(4,6,34,35)$. A study by the European group (COOS) done on 1702 patients found that there was 5-year OS of $63.3 \%$ and an EFS of 52.8\% (34). A study from the Rizzoli Institute describes patients with localized disease at initial presentation with 10 -year overall and EFS of 70 and $59 \%$, respectively (4). 
Survival rates are considerably lower in metastatic disease, having an average of $10-40 \%$ patients alive long term. Meyers et al. (36) reported that only $11 \%$ of 64 patients survived, with an average length of 20 months. Kager et al. (37) described a 5 -year overall and EFS in 202 patients of 29 and $18 \%$, respectively. Similar reports have been done by European groups with $16 \%$ rates $(n=45)$ and by American groups with $53 \%$ rates $(n=30)$, the latter study having important differences, such as a higher proportion of patients with unique lung metastases and excluding patients with irresectable disease $(38,39)$. In Peru, there are no previous reports about survival in osteosarcoma patients. Our results showed overall and EFS rates comparable to studies in developing countries (40-42).

The present study has its main limitation on the small number of patients. Similarly, there is missing information in some of the cases, due to its retrospective design, which encourage us to standardize and improve clinical records in our institution.

Nevertheless, its main strength is that it represents a first analysis of its kind in the medical literature of our country and blazes a trail for more ambitious and wider ranging future studies.

\section{REFERENCES}

1. Rosen G, Marcove RC, Caparros B, Nirenberg A, Kosloff C, Huvos AG. Primary osteogenic sarcoma: the rationale for preoperative chemotherapy and delayed surgery. Cancer (1979) 43:2163-77. doi:10.1002/10970142(197906)43:6<2163::AID-CNCR2820430602>3.0.CO;2-S

2. Jaffe N, Robertson R, Takaue Y, Cangir A, Wallace S, Carrasco H, et al. Control of primary osteosarcoma with chemotherapy. Cancer (1985) 56:461-6. doi:10.1002/1097-0142(19850801)56:3<461::AID-CNCR2820560308>3.0.CO;2-L

3. Link MP, Goorin AM, Miser AW, Green AA, Pratt CB, Belasco JB, et al. The effect of adjuvant chemotherapy on relapse-free survival in patients with osteosarcoma of the extremity. N Engl J Med (1986) 314:1600-6. doi:10.1056/ NEJM198606193142502

4. Bacci G, Ferrari S, Bertoni F, Ruggieri P, Picci P, Longhi A, et al. Long-term outcome for patients with nonmetastatic osteosarcoma of the extremity treated at the Istituto Ortopedico Rizzoli according to the Istituto Ortopedico Rizzoli/osteosarcoma-2 protocol: an updated report. J Clin Oncol (2000) 18:4016-27.

5. Petrilli AS, de Camargo B, Filho VO, Bruniera P, Brunetto AL, Jesus-Garcia R, et al. Results of the Brazilian Osteosarcoma Treatment Group Studies III and IV: prognostic factors and impact on survival. J Clin Oncol (2006) 24:1161-8. doi:10.1200/JCO.2005.03.5352

6. Meyers PA, Gorlick R, Heller G, Casper E, Lane J, Huvos AG, et al. Intensification of preoperative chemotherapy for osteogenic sarcoma: results of the Memorial Sloan-Kettering (T12) protocol. J Clin Oncol (1998) 16:2452-8.

7. Bramwell VH, Burgers M, Sneath R, Souhami R, van Oosterom AT, Voute PA, et al. A comparison of two short intensive adjuvant chemotherapy regimens in operable osteosarcoma of limbs in children and young adults: the first study of the European Osteosarcoma Intergroup. J Clin Oncol (1992) 10:1579-91.

8. Kalifa C, Razafindrakoto H, Vassal G, Contesso G, Vanel D, Edeline V, et al. Chemotherapy in osteogenic sarcoma: the experience of the Pediatric Department of the Gustave Roussy Institute. Cancer Treat Res (1993) 62:347-9. doi:10.1007/978-1-4615-3518-8_42

9. Meyers PA, Heller G, Healey J, Huvos A, Lane J, Marcove R, et al. Chemotherapy for nonmetastatic osteogenic sarcoma: the Memorial Sloan-Kettering experience. J Clin Oncol (1992) 10:5-15.

10. Winkler K, Beron G, Kotz R, Salzer-Kuntschik M, Beck J, Beck W, et al. Neoadjuvant chemotherapy for osteogenic sarcoma: results of a Cooperative German/Austrian Study. J Clin Oncol (1984) 2:617-24.

11. Goorin AM, Perez-Atayde A, Gebhardt M, Andersen JW, Wilkinson RH, Delorey MJ, et al. Weekly high-dose methotrexate and doxorubicin for

\section{CONCLUSION}

The presence of initial serum elevated AP levels and a poor histological response after neoadjuvant chemotherapy were significant predictors in children with osteosarcoma of extremities. Therefore, it reveals the need for cooperative studies that outline strategies on the basis of risk factors. It is necessary to optimize staging and intensification of treatment to improve survival rates, especially among patients with metastases at initial presentation.

\section{AUTHOR CONTRIBUTIONS}

FT, MO, and GP actively contributed to collect data and statistical process. JG, LS, and JS have reviewed and made corrections to the final version of the manuscript. All authors made important contributions to the conception or design and analysis of the work; revising it critically and made a final approval of the version to be published with agreement with the accuracy and integrity of the information on the manuscript.

osteosarcoma: the Dana-Farber Cancer Institute/the Children's Hospitalstudy III. J Clin Oncol (1987) 5:1178-84.

12. Winkler K, Beron G, Delling G, Heise U, Kabisch H, Purfürst C, et al. Neoadjuvant chemotherapy for osteogenic sarcoma: results of a randomized cooperative trial (COSS-82) with salvage chemotherapy based on histological tumor response. J Clin Oncol (1988) 6:329-37.

13. Fuchs N, Bielack SS, Epler D, Bieling P, Delling G, Körholz D, et al. Longterm results of the co-operative German-Austrian-Swiss osteosarcoma study group's protocol COSS-86 of intensive multidrug chemotherapy and surgery for osteosarcoma of the limbs. Ann Oncol (1998) 9:893-9. doi:10.1 023/A:1008391103132

14. Picci P, Bacci G, Campanacci M, Gasparini M, Pilotti S, Cerasoli S, et al. Histologic evaluation of necrosis in osteosarcoma induced by chemotherapy. Regional mapping of viable and nonviable tumor. Cancer (1985) 56:1515-21. doi:10.1002/1097-0142(19851001)56:7<1515::AID-CNCR2820560707>3.0.CO;2-6

15. Bacci G, Ferrari S, Mercuri M, Longhi A, Capanna R, Tienghi A, et al. Neoadjuvant chemotherapy for extremity osteosarcoma - preliminary results of the Rizzoli's 4th study. Acta Oncol (1998) 37:41-8. doi:10.1080/028418698423168

16. Bramer JA, van Linge JH, Grimer RJ, Scholten RJ. Prognostic factors in localized extremity osteosarcoma: a systematic review. Eur J Surg Oncol (2009) 35:1030-6. doi:10.1016/j.ejso.2009.01.011

17. Ozger H, Eralp L, Atalar AC, Toker B, Ayan I, Kebudi R, et al. [Survival analysis and the effects of prognostic factors in patients treated for osteosarcoma]. Acta Orthop Traumatol Turc (2007) 41:211-9. doi:10.3944/aott.v41i3.2559

18. Philip T, Iliescu C, Demaille MC, Pacquement H, Gentet JC, Krakowski I, et al. High-dose methotrexate and HELP [Holoxan (ifosfamide), eldesine (vindesine), platinum] - doxorubicin in non-metastatic osteosarcoma of the extremity: a French multicentre pilot study. Federation Nationale des Centres de Lutte contre le Cancer and Societe Francaise d'Oncologie Pediatrique. Ann Oncol (1999) 10:1065-71.

19. Provisor AJ, Ettiger LJ, Nachman J. Betal treatment of nonmetastatic osteosarcoma of the extremity with preoperative and postoperative chemotherapy. A report from the Children's Cancer Group. J Clin Oncol (1997) 15:76-84.

20. Petrilli AS, Gentil FC, Epelman S, Lopes LF, Bianchi A, Lopes A, et al. Increased survival, limb preservation, and prognostic factors for osteosarcoma. Cancer (1991) 68:733-7. doi:10.1002/1097-0142(19910815)68:4<733::AID-CNCR2820680412>3.0.CO;2-0

21. Petrilli S, Penna V, Lopes A, Figueiredo MT, Gentil FC. IIB osteosarcoma. Current management, local control, and survival statistics - Sao Paulo, Brazil. Clin Orthop Relat Res (1991) 270:60-6. 
22. Xie L, Guo W, Li Y, Ji T, Sun X. Pathologic fracture does not influence local recurrence and survival in high-grade extremity osteosarcoma with adequate surgical margins. J Surg Oncol (2012) 106:820-5. doi:10.1002/jso.23150

23. Berner K, Hall KS, Monge OR, Weedon-Fekjær H, Zaikova O, Bruland ØS. Prognostic factors and treatment results of high-grade osteosarcoma in Norway: a scope beyond the "classical" patient. Sarcoma (2015) 2015:516843. doi:10.1155/2015/516843

24. Saeter G, Wiebe T, Wiklund T, Monge O, Wahlqvist Y, Engström K, et al. Chemotherapy in osteosarcoma. The Scandinavian Sarcoma Group experience. Acta Orthop Scand Suppl (1999) 285:74-82.

25. Taylor WF, Ivins JC, Unni KK, Beabout JW, Golenzer HJ, Black LE. Prognostic variables in osteosarcoma: a multi-institutional study. J Natl Cancer Inst (1989) 81:21-30. doi:10.1093/jnci/81.1.21

26. Bacci G, Ferrari S, Longhi A, Perin S, Forni C, Fabbri N, et al. Pattern of relapse in patients with osteosarcoma of the extremities treated with neoadjuvant chemotherapy. Eur J Cancer (2001) 37:32-8. doi:10.1016/S0959-8049(01)00229-5

27. Hudson M, Jaffe MR, Jaffe N, Ayala A, Raymond AK, Carrasco H, et al. Pediatric osteosarcoma: therapeutic strategies, results, and prognostic factors derived from a 10-year experience. J Clin Oncol (1990) 8:1988-97.

28. Bacci G, Bertoni F, Longhi A, Ferrari S, Forni C, Biagini R, et al. Neoadjuvant chemotherapy for high-grade central osteosarcoma of the extremity. Histologic response to preoperative chemotherapy correlates with histologic subtype of the tumor. Cancer (2003) 97:3068-75. doi:10.1002/cncr.11456

29. Wilkins RM, Cullen JW, Odom L, Jamroz BA, Cullen PM, Fink K, et al. Superior survival in treatment of primary nonmetastatic pediatric osteosarcoma of the extremity. Ann Surg Oncol (2003) 10:498-507. doi:10.1245/ASO.2003.03.061

30. Mavrogenis AF, Abati CN, Romagnoli C, Ruggieri P. Similar survival but better function for patients after limb salvage versus amputation for distal tibia osteosarcoma. Clin Orthop Relat Res (2012) 470:1735-48. doi:10.1007/ s11999-011-2238-7

31. Bacci G, Ferrari S, Lari S, Mercuri M, Donati D, Longhi A, et al. Osteosarcoma of the limb. Amputation or limb salvage in patients treated by neoadjuvant chemotherapy. J Bone Joint Surg Br (2002) 84:88-92. doi:10.1302/0301-620X.84B1.12211

32. Bacci G, Longhi A, Ferrari S, Briccoli A, Donati D, De Paolis M, et al. Prognostic significance of serum lactate dehydrogenase in osteosarcoma of the extremity: experience at Rizzoli on 1421 patients treated over the last 30 years. Tumori (2004) 90:478-84.

33. Bacci G, Longhi A, Versari M, Mercuri M, Briccoli A, Picci P. Prognostic factors for osteosarcoma of the extremity treated with neoadjuvant chemotherapy: 15-year experience in 789 patients treated at a single institution. Cancer (2006) 106:1154-61. doi:10.1002/cncr.21724

34. Bielack SS, Kempf-Bielack B, Delling G, Exner GU, Flege S, Helmke K, et al. Prognostic factors in high-grade osteosarcoma of the extremities or trunk: an analysis of 1,702 patients treated on neoadjuvant cooperative osteosarcoma study group protocols. J Clin Oncol (2002) 20:776-90. doi:10.1200/ JCO.20.3.776

35. Meyers PA, Schwartz CL, Krailo M, Kleinerman ES, Betcher D, Bernstein ML, et al. Osteosarcoma: a randomized, prospective trial of the addition of ifosfamide and/or muramyl tripeptide to cisplatin, doxorubicin, and high-dose methotrexate. J Clin Oncol (2005) 23:2004-11. doi:10.1200/JCO.2005.06.031

36. Meyers PA, Heller G, Healey JH, Huvos A, Applewhite A, Sun M, et al. Osteogenic sarcoma with clinically detectable metastasis at initial presentation. J Clin Oncol (1993) 11:449-53.

37. Kager L, Zoubek A, Pötschger U, Kastner U, Flege S, Kempf-Bielack B, et al. Primary metastatic osteosarcoma: presentation and outcome of patients treated on neoadjuvant Cooperative Osteosarcoma Study Group protocols. $J$ Clin Oncol (2003) 21:2011-8. doi:10.1200/JCO.2003.08.132

38. Voute PA, Souhami RL, Nooij M, Somers R, Cortés-Funes H, van der Eijken JW, et al. A phase II study of cisplatin, ifosfamide and doxorubicin in operable primary, axial skeletal and metastatic osteosarcoma. European Osteosarcoma Intergroup (EOI). Ann Oncol (1999) 10:1211-8. doi:10.102 3/A:1008361612767

39. Harris MB, Gieser P, Goorin AM, Ayala A, Shochat SJ, Ferguson WS, et al. Treatment of metastatic osteosarcoma at diagnosis: a Pediatric Oncology Group Study. J Clin Oncol (1998) 16:3641-8.

40. Faisham WI, Mat Saad AZ, Alsaigh LN, Nor Azman MZ, Kamarul Imran M, Biswal BM, et al. Prognostic factors and survival rate of osteosarcoma: a single-institution study. Asia Pac J Clin Oncol (2015). doi:10.1111/ajco.12346

41. Râmyza F, de Sousa L, Sá Lopes J, Barbosa M. Evaluation of prognostic factors and survival among patients with osteosarcoma attended at a philanthropic hospital in Teresina, Piauí, Brazil. Rev Bras Ortop (2013) 48(1):87-91. doi:10.1016/j.rboe.2012.05.002

42. Rech A, Castro CG Jr, Mattei J, Gregianin L, Di Leone L, David A, et al. [Clinical features in osteosarcoma and prognostic implications]. J Pediatr (Rio J) (2004) 80:65-70. doi:10.2223/1136

Conflict of Interest Statement: The authors declare that the research was conducted in the absence of any commercial or financial relationships that could be construed as a potential conflict of interest.

Copyright (C) 2016 Vasquez, Tarrillo, Oscanoa, Maza, Geronimo, Paredes, Silva and Sialer. This is an open-access article distributed under the terms of the Creative Commons Attribution License (CC BY). The use, distribution or reproduction in other forums is permitted, provided the original author(s) or licensor are credited and that the original publication in this journal is cited, in accordance with accepted academic practice. No use, distribution or reproduction is permitted which does not comply with these terms. 